\title{
HISTOLOGICAL AND IMMUNOHISTOCHEMICAL EVALUATION OF THE AMELIORATING ROLE OF PROPOLIS AND/OR INTERMITTENT FASTING ON INDUCED ORAL MUCOSITIS IN ALBINO RATS
}

\author{
Rania Ahmed Awwad*
}

\begin{abstract}
Context: Oral Mucositis is a multi-etiological inflammatory condition, which's very painful and complicating patient's life, hence it was appealing to find natural remedies, dietary modifications or both to manage this condition successfully.

Aim: to evaluate histologically and immunohistochemically the efficiency of Propolis and / or Intermittent Fasting in ameliorating Oral Mucositis.

Materials \& Methods: Forty male Albino rats weighing 200-250 grams were divided into: Negative Control Group (-ve control Gp): eight rats kept healthy, Positive Control Group (+ve): eight rats subjected to Oral Mucositis (OM) induction in their buccal mucosa, Propolis (P) $G p$ : eight rats subjected to OM Induction, given daily $100 \mathrm{mg} / \mathrm{kg}$ Propolis orally, Intermittent Fasting (IF) Gp: eight rats subjected to OM induction and IF for 16 hours, Propolis \& Intermittent Fasting $(\boldsymbol{P} \& \boldsymbol{I F}) \boldsymbol{G} \boldsymbol{p}$ : eight rats subjected to OM induction and maintained on both P (daily $100 \mathrm{mg} / \mathrm{kg}$ orally) and IF simultaneously. After three weeks, the buccal mucosae of all groups were processed and stained by H\&E, Masson's Trichrome, and Anti-PCNA then examined by light microscope.
\end{abstract}

Results: the +ve control Gp showed statistically significant increase in number of vacuolated epithelial cells, apparent decrease in immature collagen fibers within connective tissue (CT) and statistically significant reduction in PCNA positive cells. In the P, IF, and P\&IF Gps, there were marked improvement in epithelium and CT. The (P\&IF) Gp manifested the maximum decrease in vacuolated epithelial cells, greater amount of immature collagen fibers, and maximum increase in PCNA positive cells.

Discussion \& conclusion: OM caused tissue destruction secondary to the inflammatory state leading to elevated levels of reactive oxygen species, which was evident in the +ve control Gp. Propolis intake greatly improved the histological appearance (confirming anti-inflammatory role of Propolis) as well as Intermittent Fasting (confirming immune-boosting action). However, implementing both $\mathrm{P} \&$ IF treatment protocols resulted in maximum improvement due to combined anti-inflammatory, anti-oxidant, immune-enhancing actions of both $\mathrm{P}$ and IF.

KEY WORDS: Oral Mucositis, Propolis, Intermittent Fasting.

\footnotetext{
* Lecturer of Oral biology, Faculty of Dentistry, Ain Shams University.
} 


\section{INTRODUCTION}

Oral wellbeing is significant for a sound life-style of persons of all ages. Oral lesions cause distress or soreness that could impede person's daily actions as mastication, deglutition \& speech which may later generate symptoms such as xerostomia, halitosis, or other oral pathosis ${ }^{[1]}$.

Oral mucosal lesions were described as any alteration in oral mucosal surface that may appear as white, red, ulcerative and pigmented lesions, or any swelling. There are numerous etiological factors for oral mucosal lesions such as infection (viruses, bacteria, parasites, and fungi), chemical and thermal factors, systemic diseases, trauma, neoplasia, immunological factors, and persistent unhealthy behaviors such as tobacco and alcohol consumption ${ }^{[2]}$.

However, Oral Mucositis (OM) can be defined as an inflammatory mucosal disruption resulting from various factors as chemotherapy and/or radiation therapy, which in severe cases can impair patients' quality of life. Moreover, OM can also occur due to mucosal infection or systemic involvement resulting from compromised immunity ${ }^{[3]}$. Mucositis might occur in more than $98 \%$ of bone-marrowtransplant patients. It is the most prevalent and grave side effect reported in the first three months after transplantation ${ }^{[4,5]}$. Radiotherapy-induced OM causes vast dose-limiting symptoms in patients with head and neck malignant neoplasms. This offers a major challenge for radiotherapy specialists as it causes interruption of cancer treatment, reduced local tumor management, and alteration in dose fractionation ${ }^{[6]}$.

During menopause, women pass through biological and endocrine alterations, directly involving oral tissues. The burning sensation in normal oral mucosa is known as "burning mouth syndrome" is frequently encountered in postmenopausal ladies ${ }^{[7]}$.

Wound recovery process involves a complex series of numerous cellular and biochemical actions leading to tissue repair. During wound healing, wound proceeds through three stages: inflammation, proliferation, and maturation ${ }^{[8]}$. Recently, when steroids and other drugs, inflicting many adverse effects, are used to treat various oral lesions, researchers are investigating other treatment modalities which have equal efficiency and minimum or no side-effects. Extensive assortment of therapeutic agents is accessible to manage OM. Presently, many herbal medications are becoming trendy in managing this condition due to minimal side effects than artificial drugs ${ }^{[9]}$. For example, Aloe Vera gel has numerous pharmacological effects including anti-inflammatory, antioxidant, bactericidal, fungicidal, anti-tumor, hypoglycemic and immune boosting effects ${ }^{[1,9]}$. Aloe Vera gel had also been used in dentistry and showed good results. ${ }^{[10]}$.

Honey, in some recently published researches appeared to possess a curative effect on oral mucositis ${ }^{[11,12]}$. It is well-established that Bee products are rich sources of natural antioxidants, mainly flavonoids and phenolic acids. Propolis, which is also commonly referred to as "Bee Glue," is a sticky mixture that honey bees made by blending their saliva (having particular enzymes) and beeswax with exudate collected mostly from leaves and flowers, and barks of many tree species. The word propolis originated from two Greek words pro and polis, which mean "protection" and "city" or "society," respectively. Bees used it chiefly as a sealant and a sanitizing material. They used it for sealing pits and fissures, smoothing inner surfaces, and maintaining internal temperature of their beehive ${ }^{[13,14,15]}$.

The antioxidant potentials of propolis were established in many studies and were found to be comparable to the man-made antioxidants ${ }^{[16]}$. Moreover, the biological properties of propolis surpassed antioxidant activity to include also, antimicrobial, anti-inflammatory, and cytotoxic effects. Meanwhile, many researches were done to assess the antiparasitic potential of propolis ${ }^{[16,17,18]}$. 
Dietary restrictions, particularly Intermittent Fasting (IF), offers a non-pharmacological method to enhance cellular abilities for endogenous protective mechanisms to resist neuro-degenerative diseases and aging-associated alterations [19, 20]. Also, it was found that protein degradation through the autophagy-lysosomal system is boosted in many tissues by IF ${ }^{[21,22,23]}$. Meanwhile, it is well established that IF improves tissue ability to resist senility disorders and extends life span [24, 25]. Therefore, it should be mentioned that almost all major religions encouraged or even instructed their followers to go through intermittent fasting on a regular base, in order to enjoy a healthy life.

Thus, it was appealing to evaluate the ameliorating effects of propolis and/or Intermittent fasting, on oral mucositis in rat model.

\section{MATERIALS AND METHODS}

\section{Animals:}

This research was reviewed and approved by the "Research Ethics Committee" of Faculty of Dentistry, Ain Shams University (Approval number: FDASU - REC IR 101901). In this study, forty adult male Albino rats weighing between 200250 grams were used. The rats were housed in separate cages, eight rats per cage, in the Animal House of the Medical Research Center of Ain Shams University. They were maintained under controlled conditions of temperature $\left(25 \pm 2^{\circ} \mathrm{C}\right)$, humidity $(50 \pm 5 \%)$, and light (14 and $10 \mathrm{~h}$ of light and dark, respectively). Animals were provided with food and water ad libitum. Animals were kept under constant supervision of specialized veterinarian throughout the whole experiment.

\section{Materials:}

- Propolis resin extract $100 \mathrm{mg} / \mathrm{ml}$, (Nature's Answer, USA) was used in this study, in a daily dose of $100 \mathrm{mg} / \mathrm{kg}$ in $1 \mathrm{ml}$ of distilled water, administered orally by sterile oral feeding tube ${ }^{[26]}$.
- Primary antibody (mouse monoclonal AntiPCNA antibody, Clone PC10, cat. \# sc-56) was purchased from (Santa Cruz Biotechnology, Inc. USA), Ultra-Sensitive avidin-biotin peroxidase complex mouse IgG staining kit (32052) was delivered by (Thermo Fisher Scientific Inc., Waltham, MA, USA).

- Masson's Trichrome special stain (HT15-Sigma Aldrich).

\section{Study Design:}

Rats were divided into five groups, eight rats each, as follows:

1. Negative Control (-ve control) Group: In which eight rats were kept in a normal, healthy state throughout the experiment, and fed ad libitum for three consecutive weeks.

Rats of the remaining groups were subjected to mechanical injury of the buccal mucosa (by careful scratching using sterile lancet), followed by $5 \%$ acetic acid application to induce oral ulceration ${ }^{[27]}$.

1. Positive Control (+ve control) Group: In which eight rats were subjected to induced oral ulcerations. They received food ad libitum for three consecutive weeks.

2. Propolis (P) Group: In which eight rats were subjected to induced oral ulcerations. Rats of this group also received Propolis extract orally (by sterile oral feeding tube) at a daily dose of $100 \mathrm{mg} / \mathrm{kg}$ in $1 \mathrm{ml}$ of distilled water ${ }^{[26]}$, for three consecutive weeks. They received food $a d$ libitum.

3. Intermittent Fasting (IF) Group: In which eight rats were subjected to induced oral ulcerations. Rats of this group were also subjected to IF for 16 hours' food and water deprivation, daily for three consecutive weeks ${ }^{[28,29]}$.

4. Propolis + Intermittent Fasting (P\&IF) Group: In which eight rats were subjected to 
induced oral ulcerations. Rats of this group were given Propolis extract orally at a daily dose of $100 \mathrm{mg} / \mathrm{kg}$ in $1 \mathrm{ml}$ of distilled water, and at the same time, they were subjected to IF as previously mentioned, daily for three consecutive weeks.

\section{Sample preparation}

After three weeks, rats were sacrificed by a high dose of anesthesia at the end of the experiment, the buccal mucosa of all rats was excised and instantly fixed in $10 \%$ phosphate buffered formaldehyde solution for 48 hours. Specimens were then appropriately rinsed under running water then dehydrated by transmitting them through rising concentrations of alcohol. The specimens were transmitted to xylene to be cleared from alcohol then impregnated in paraffin and embedded in the paraffin wax blocks. Four $\mu \mathrm{m}$-thick sections were then produced and put in xylene and transmitted in reducing concentrations of alcohol then distilled water for removal of paraffin wax ${ }^{[30]}$. The sections were finally stained by Hematoxylin \& Eosin (H\&E), Masson's Trichrome special stain (for collagen demarcation), and Antiproliferating Cell Nuclear Antigen (Anti-PCNA) which is a marker for cell regeneration.

\section{Masson's Trichrome Staining Technique}

This method is used for the detection of collagen and to discriminate between mature and immature collagen fibers in tissues, on formalin-fixed, paraffin-embedded sections. The immature collagen fibers will be stained blue while the mature ones will display varying hues of red.

\section{Procedure:}

1. Sections were deparaffinized and rehydrated through 100\% alcohol, 95\% alcohol $70 \%$ alcohol.

2. Section were then washed in distilled water.

3. They were re-fixed in Bouin's solution for 1 hour at $56 \mathrm{C}$ to improve staining quality.
4. Sections were washed under running tap water for 5-10 minutes to eliminate yellow coloration.

3. They were stained in Weigert's iron hematoxylin working solution for 10 minutes.

4. Then, they were rinsed in running warm tap water for 10 minutes.

5. Sections were washed in distilled water.

6. They were stained in Biebrich Scarlet-Acid Fuchsin solution for 10-15 minutes.

7. Then, washed in distilled water.

8. They were differentiated in PhosphomolybdicPhosphotungstic acid solution for 10-15 minutes.

9. Section were then transferred directly (without rinse) to Aniline Blue solution and stain for 5-10 minutes. They were then rinsed briefly in distilled water and differentiated in $1 \%$ acetic acid solution for 2-5 minutes.

10. They were washed in distilled water.

11. Then, dehydrated very quickly through $95 \%$ ethyl alcohol, absolute ethyl alcohol to wipe off Biebrich Scarlet-Acid Fuchsin staining and then, cleared in xylene.

12. Section were finally mounted with resinous mounting medium ${ }^{[30]}$.

Expected Results: Immature collagen appears blue while mature collagen appears red or reddish blue.

\section{Immunohistochemical staining using Anti-PCNA}

$4 \mu \mathrm{m}$ sections were placed over positively charged slides, then placed in $65^{\circ} \mathrm{C}$ oven for an hour. The sections were deparaffinized and rehydrated, washed and submerged in Tris buffered solution (20 mM Tris- $\mathrm{HCl}, 150 \mathrm{mM} \mathrm{NaCl}, \mathrm{pH}$ 7.4) for $\mathrm{pH}$ adjustment. Sections were then incubated in $0.3 \%$ hydrogen peroxide $\left(\mathrm{H}_{2} \mathrm{O}_{2}\right)$ at room temperature for 30 minutes to inhibit the activity of endogenous peroxidase. Then, slides were submerged in blocking solution (normal goat serum) at room 
temperature for 30 minutes to help antigen recovery and eliminate non- specific background staining, and then incubated with primary anti- body (mouse monoclonal Anti-PCNA antibody, Clone PC10) at room temperature for 60 minutes. The sections were washed by PBS (0.1 M phosphate, $0.15 \mathrm{M} \mathrm{NaCl}$; $\mathrm{pH}$ 7.5) and incubated with secondary biotinylated antibody (Biotinylated horse anti-mouse IgG) at room temperature in a humidity chamber for an hour, then incubated with avidin-biotin horseradish peroxidase complex for 30 minutes. The color reaction was developed when adding $\mathrm{DAB}$ solution $\left(0.5 \mathrm{mg} / \mathrm{ml} \mathrm{DAB}\right.$ and $\left.0.1 \% \mathrm{H}_{2} \mathrm{O}_{2}\right)$ to the sections for 10 minutes, after that, rinsed with distilled water. The sections were counterstained by hematoxylin for 2 minutes, dehydrated in graded alcohol and cleared by xylene. Eventually, cover slips were placed and slides were examined under light microscope ${ }^{[31]}$.

\section{Histomorphometric Analysis:}

Image J software (Version 1.41a, NIH, USA) was used for histo-morphometric analysis, in the Precision Measurement Unit, Oral Pathology Department, Faculty of Dentistry, Ain Shams University. Images of Anti-PCNA immune stained sections, were analyzed by the software to count the number of immune-positive cells per unit area in the epithelium of the studied specimens. Also, the same software was used to count the number of vacuolated epithelial cells. Three representative specimens were chosen from each group. Three representative fields were assessed/specimen to display the whole epithelium, considerable area from the underlying C.T, as well as proper Anti-PCNA reaction. Fields were captured by a digital camera (Canon DSLR EOS 1200D, Japan) which was mounted on a light microscope (BX60, Olympus, Japan). The obtained images were transferred to the computer system for analysis ${ }^{[31]}$.

\section{Statistical analysis}

Numerical data of mean number of Anti-PCNA positive cells, and mean number of vacuolated epithelial cells were examined for normality by monitoring the distribution of data and using tests of normality. One-way ANOVA test was performed to compare between the five groups. When ANOVA test was significant, Bonferroni's post-hoc test was done for pair-wise comparisons between each two groups. The significance level was set at $\mathrm{P} \leq 0.05$. Statistical analysis was performed using IBM ${ }^{\circledR}$ SPSS $®$ Statistics Version 21 for Windows.

\section{RESULTS}

\section{Light microscopic results}

\section{Hematoxylin \& Eosin $(H \& E)$}

\section{Negative Control Group (Gp)}

Examination of H\&E stained sections of the buccal mucosa of this Gp showed normal appearance of ortho-keratinized stratified squamous epithelium, covered by well-formed continuous keratin layer. Intraepithelial cell vacuolations were rarely encountered. The underlying connective tissue (CT) showed normal histological structure. Spindleshaped fibroblasts with basophilic cytoplasm were regularly arranged \& intermingled with well-formed collagen fiber bundles. Very few inflammatory cells were detected close to blood vessels. (Fig.1a)

\section{Positive Control Gp:}

In this $\mathrm{Gp}$, disrupted epithelial layer with detached, thin and discontinuous keratin layer was seen in some areas demarcating the ulcerated regions, some areas of CT showed hyalinization of collagen fibers. (Fig. 1b). Many epithelial cells displayed intracytoplasmic cell-vacuolations, with displacement of the nuclei towards one side in some of them (Fig 1c). The underlying CT showed marked inflammatory cell infiltration, dilated engorged blood vessels and extracellular edema. (Fig 1d). 


\section{Propolis Gp:}

The epithelial layer of this group showed almost normal histological appearance resembling that of -ve control Gp, with continuous keratin layer of even thickness. Intracytoplasmic cell vacuolations were rarely detected within the epithelium of this $\mathrm{Gp}$, in most specimens. The CT presented an appearance similar to the -ve control Gp, with marked reduction in the presence of inflammatory cells. Well-developed endothelially lined blood vessels and well-formed collagen fibers were also seen in the CT of this Gp (Fig. 1e).

\section{Intermittent Fasting Gp:}

The epithelial layer of this $\mathrm{Gp}$ also showed almost normal histological structure with thick continuous keratin layer, except in some few regions in which the epithelium was reduced in thickness and in its keratin coverage. Intracytoplasmic cell vacuolations were rarely spotted in this Gp. CT of this Gp. showed almost normal histological appearance, similar to -ve control Gp. Few inflammatory cells were seen near the blood vessels. No extracellular edema was seen in the CT (Fig.1f).

\section{P\&IF Gp:}

The histological appearance of this Gp greatly resembled the -ve control group as the epithelium was thick and uniform, rare intracytoplasmic vacuolations, and thick continuous keratin layer. CT appeared similar to the -ve control group, with rare inflammatory cells, blood vessels of variable diameters were detected, lined by endothelial cells, resembled those of the -ve control Gp, no extracellular edema, and abundant well-formed collagen fibers were evident, (Fig.1g).

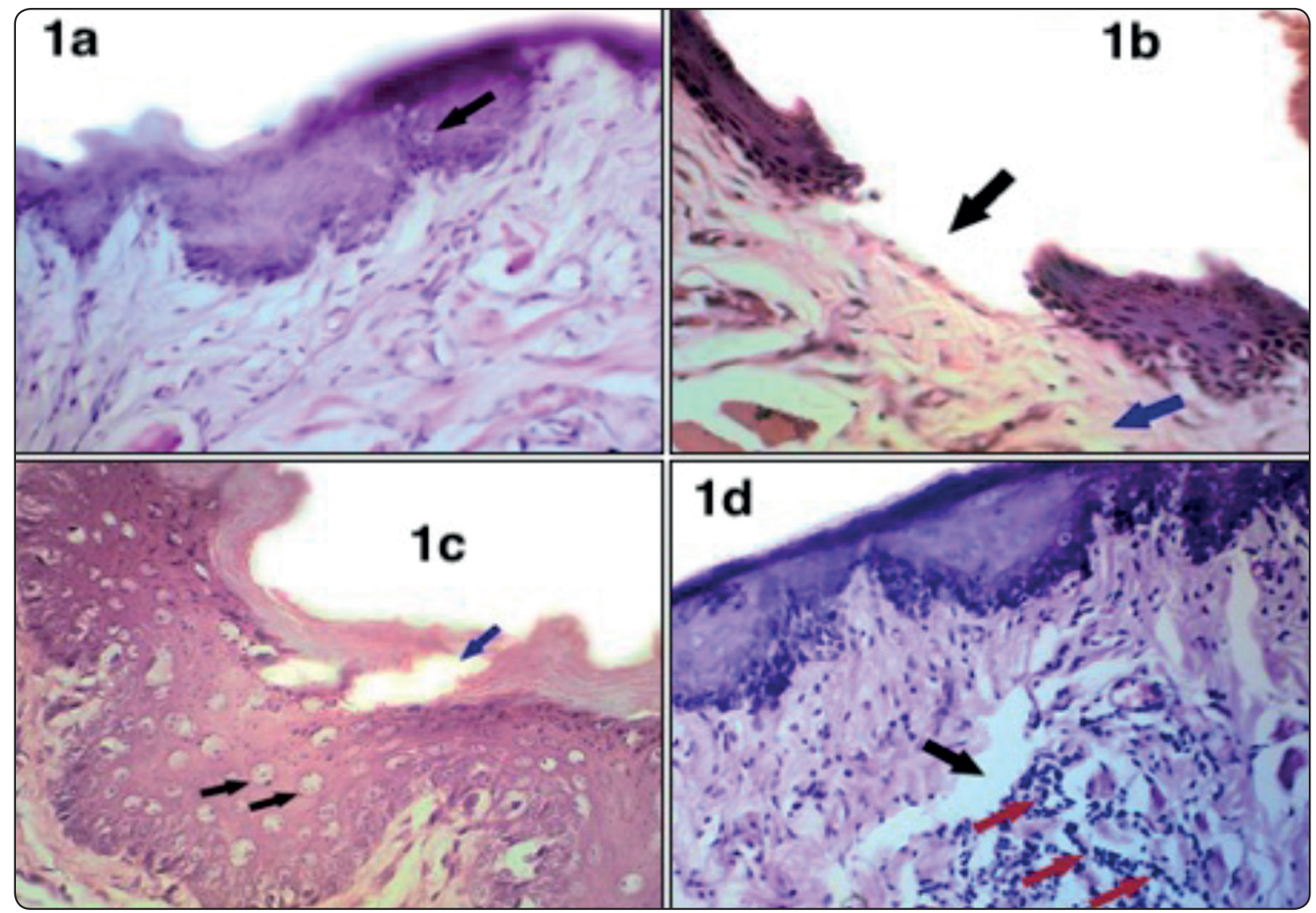




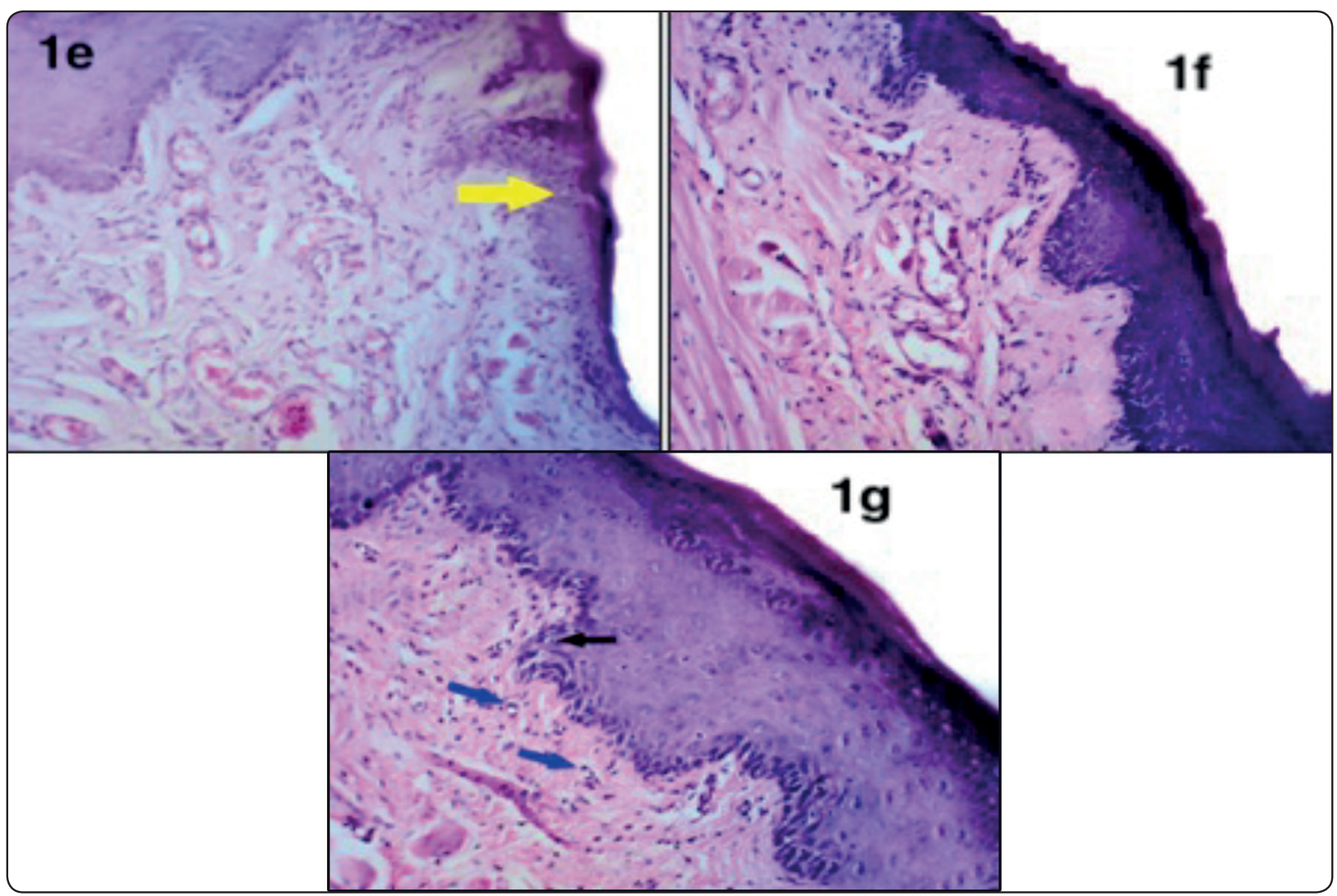

Fig. (1) Photomicrographs of buccal mucosa of a rat of: -ve control Gp in 1a, showing : keratinized stratified squamous epithelium with rare intraepithelial vacuolations (arrow), well developed CT with irregular bundles of collagen fibers and well-formed blood vessels; +ve control Gp in 1b, showing : discontinuity of epithelium and keratin, demarcating remnants of the ulcerated region (black arrow). Hyalinization of collagen fibers was seen in some areas (blue arrow), also in 1c of the same $\mathrm{Gp}$, there was keratin detachment (blue arrow) and numerous intracytoplasmic cell vacuolations in the epithelium (black arrows). In $1 \mathrm{~d}$ of the same $\mathrm{Gp}$, there was marked extracellular edema in CT (black arrow), inflammatory cell infiltration probably lymphocytes (red arrows) near dilated engorged blood vessels; Propolis Gp in 1e: showed histological structure similar to -ve control Gp with few intracytoplasmic vacuolations in the epithelium (arrow). CT displayed normal image as -ve control Gp; Intermittent Fasting Gp in 1f: showed epithelium and CT image similar to -ve control Gp; P\&IF Gp in 1g: showed histological structure of epithelium \& CT similar to -ve control Gp. The epithelium showed rare intracellular vacuolations (black arrow), well-formed CT with numerous blood vessels (blue arrows), (H\&E, x 400).

\section{Masson's Trichrome Staining Results}

\section{Negative Control Gp (-ve):}

Light microscopic (LM) examination of specimens stained with Masson's Trichrome special stain revealed thick stratified squamous epithelium with sound keratin layer. CT displayed intermingling of mature (red-colored) and immature (blue-colored) collagen fibers. Blood vessels showed normal structure, lined by endothelial cells and with patent lumina. No extracellular edema was detected, (Fig. 2a).

\section{Positive Control Gp (+ve):}

LM examination of this Gp showed thinning \& irregularity of epithelium with disruption of keratin in some areas. CT presented areas with extracellular edema. Majority of collagen fibers of the CT were stained red while very few were stained blue after staining with Masson's Trichrome. Blood vessels were dilated, (Fig. 2b).

\section{Propolis $G p(P)$ :}

LM examination of this Gp revealed epithelium of uneven thickness and CT with numerous wellformed blood vessels, some of which were dilated. Almost all collagen fibers appeared blue (immature), (Fig. 2c). 


\section{Intermittent Fasting Gp (IF):}

LM examination of this Gp presented uneven thickness of the epithelium with some intraepithelial cell vacuolations, covered by thick keratin layer. CT showed strong blue coloration of collagen fibers with rare presence of red-colored fibers. No extracellular edema was detected in the CT. Blood vessels displayed normal structure, (Fig. 2d).

\section{$P \&$ IF Gp:}

This Gp's specimens revealed thick epithelium, of even thickness. No intracytoplasmic vacuolations were detected in most specimens. CT showed numerous, normal-looking blood vessels with various diameters. No extracellular edema was seen. Collagen fibers were intensely stained blue, (Fig. 2e).

\section{Immunohistochemical Staining (using anti} PCNA) Results

Anti-PCNA immune-stain was used to detect the proliferating cells as the positive reactions revealed brown nuclear staining.

\section{-ve Control Gp:}

LM examination of this Gp specimens showed distinct positive reaction in all basal and few para-basal cells of the epithelium, some cells with positive reaction within the $\mathrm{CT}$ (Fig. 3a).

\section{+ve Control Gp:}

This Gp specimens' epithelium showed some positive reactions alternating with negative ones in basal cell layer. Minimal or rare positive reaction was detected in CT (Fig. 3b).

\section{Propolis (P) Gp:}

LM examination of this Gp specimens presented numerous positively stained cells in the basal and parabasal layers of epithelium. CT also displayed many positive cells (Fig. 3c).

\section{Intermittent Fasting (IF) Gp:}

LM examination of this group specimens revealed that almost all of the basal and many of the parabasal epithelial cells manifested positive reaction as well as some $\mathrm{CT}$ cells (Fig. 3d).

\section{P\&IF Gp:}

LM examination of this group specimens showed that majority of basal and parabasal cells of the epithelium displayed strong positive reaction as well as numerous CT cells (Fig. 3e).

\section{Statistical Results}

The results of the present study are demonstrated in the following tables and figures. 


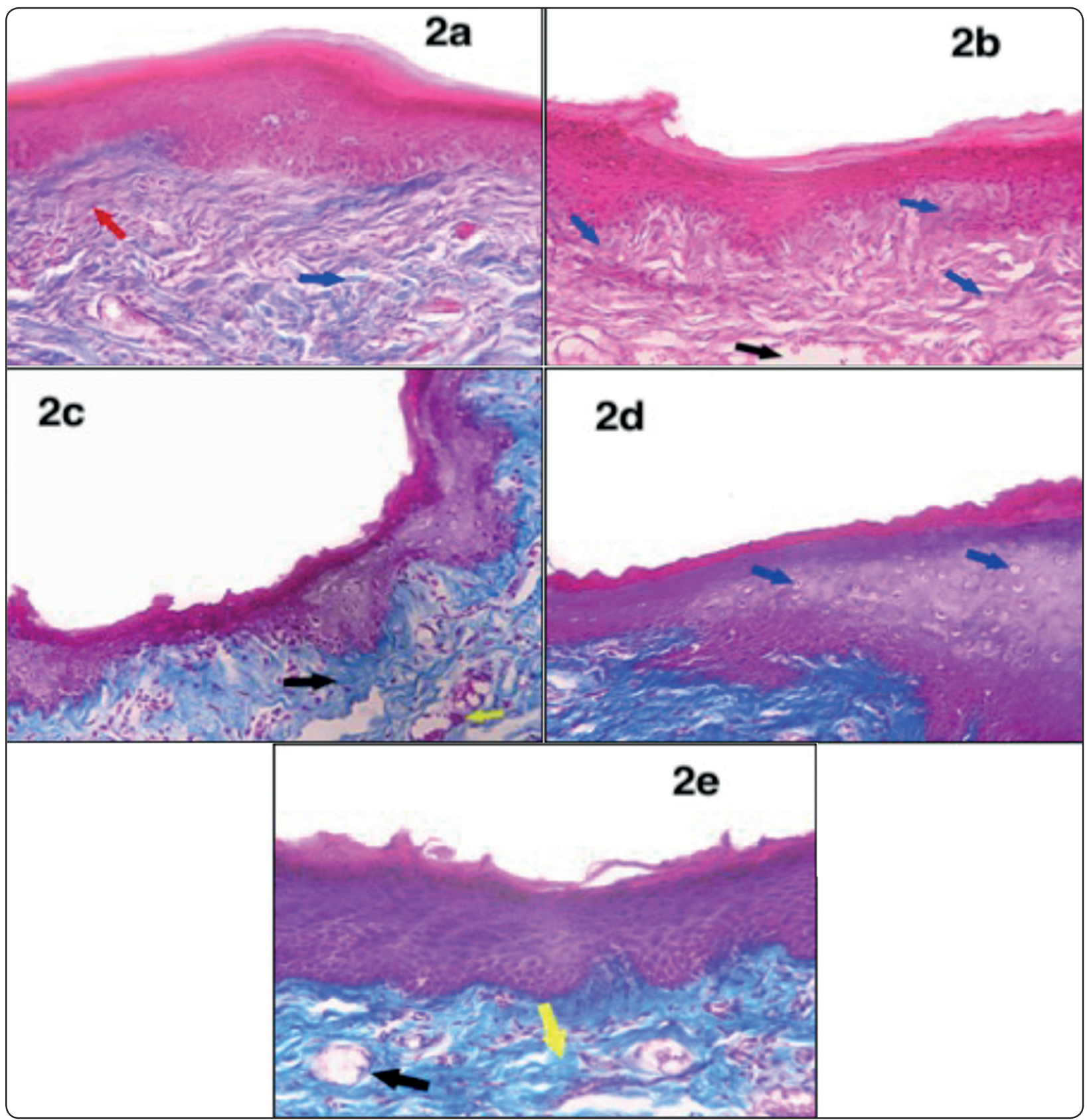

Fig. (2) Photomicrographs of buccal mucosa of a rat of : -ve control Gp in 2a, showing : apparently thick keratinized stratified epithelium, CT displayed apparently almost equal amounts of mature (red) \& immature (blue) collagen fibers; (red \& blue arrows respectively). Blood vessels displayed normal structure, CT showed no edema; +ve control Gp in $\mathbf{2 b}$, showing thin \& irregular epithelium, disrupted keratin in some areas, most of the collagen fibers were mature (red) while very few immature (blue) fibers were detected (blue arrows). CT showed extracellular edema (black arrow). Blood vessels were dilated; $\mathbf{P}$ Gp in 2c, showing uneven thickness of epithelium and CT with numerous well-formed blood vessels, some of which were dilated (yellow arrow). Apparently, all collagen fibers appeared blue (black arrow); IF Gp in 2d, showing epithelium with uneven thickness, thick keratin layer, intra-epithelial cell vacuolations were detected (arrows). CT showed strong blue coloration of collagen fibers with rare red-colored fibers. No extracellular edema was detected in the CT; P\&IF $\mathrm{Gp}$ in $\mathbf{2 e}$, showing thick epithelium of even thickness, in which no intracytoplasmic cell vacuolations were detected. CT showed numerous multi-size blood vessels (black arrow). No extracellular edema was seen. Collagen fibers were intensely stained blue (yellow arrow). (Masson's Trichrome, X 400). 

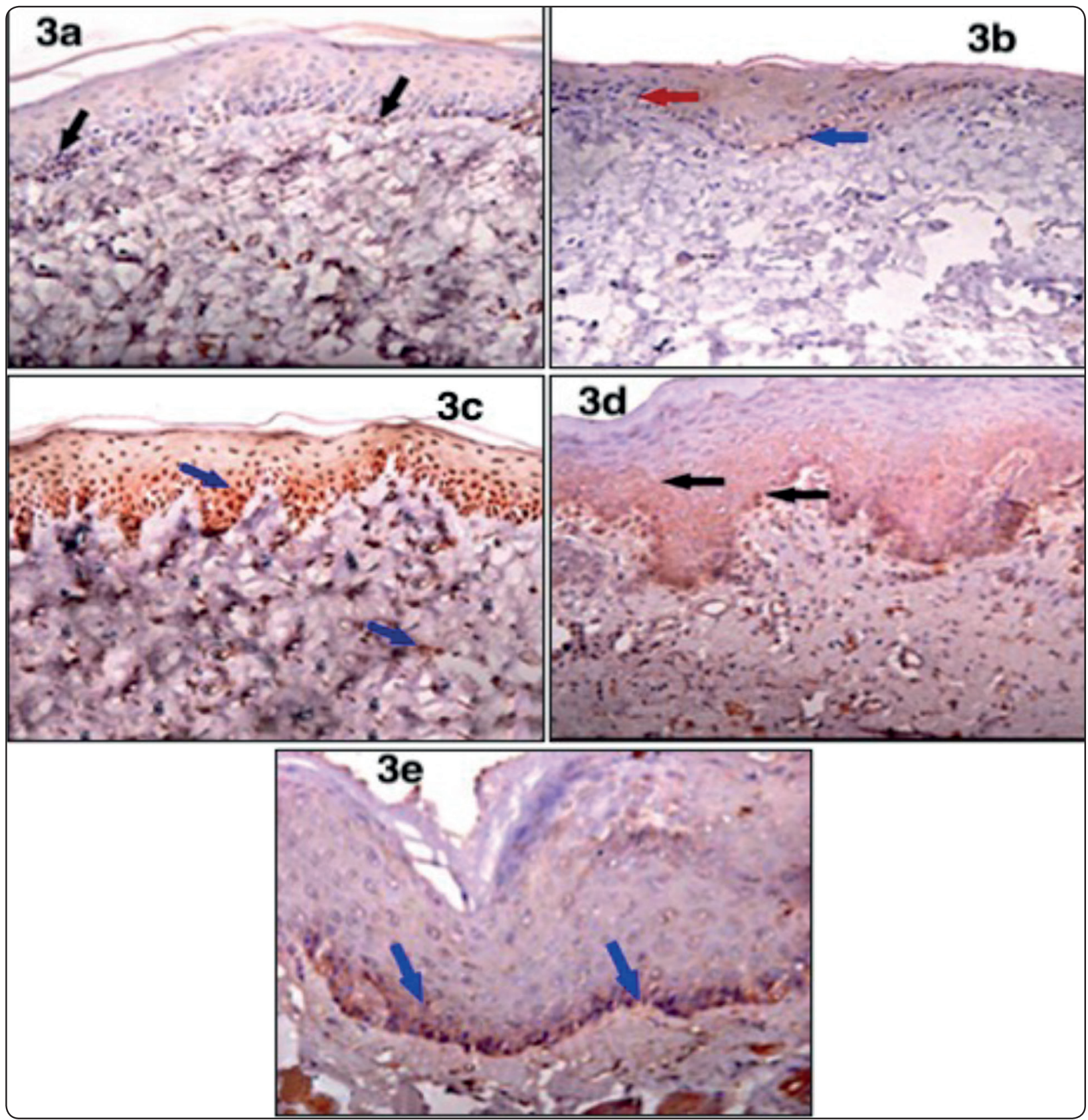

Fig. (3) Photomicrographs of buccal mucosa of a rat of : -ve control Gp in 3a, showing positive reaction in all basal and few parabasal cells of the epithelium (arrows), and some cells with positive reaction within the CT; +ve control Gp in $\mathbf{3 b}$, showing some positive reactions (blue arrow), alternating with negative ones (red arrow) in basal cell layer. Minimal or rare positive reaction was detected in CT; $\mathbf{P}$ Gp in 3c, showing positive reaction (arrows), in basal and parabasal layers of epithelium as well as CT; IF Gp in 3d, showing positive reaction (arrows), in basal and parabasal layers of epithelium. CT also displayed many positive cells; $\mathbf{P} \mathbf{\& I F}$ Gp in $\mathbf{3 e}$, showing strong positive reaction (arrows), in basal and parabasal layers of epithelium. CT also displayed many positive cells (Anti- PICNA, $x$ 400) . 
TABLE (1): Comparison between groups according to number of vacuolated epithelial cells.

\begin{tabular}{|c|c|c|c|c|c|c|c|c|}
\hline \multirow{2}{*}{ Groups } & \multicolumn{5}{|c|}{ No. of Vacuolated Epithelial Cells } & \multicolumn{3}{|c|}{ ANOVA test } \\
\hline & Min. & Max. & Mean & $\pm \mathrm{SD}$ & Change $\%$ & Bonferroni & $\mathrm{F}$ & p-value \\
\hline Control -ve Group & 4 & 23 & 13.56 & 5.64 & & --- & \multirow{5}{*}{42.255} & \multirow{5}{*}{$<0.001 * *$} \\
\hline Control +ve Group & 34 & 80 & 57.33 & 15.91 & $322.79 \%$ & $<0.001 * *$ & & \\
\hline Propolis Group & 8 & 40 & 19.44 & 9.40 & $43.36 \%$ & 0.172 & & \\
\hline Intermittent Fasting Group & 13 & 27 & 19.00 & 4.47 & $40.12 \%$ & 0.206 & & \\
\hline Propolis + Intermittent Fasting Group & 3 & 13 & 8.11 & 3.30 & $-40.19 \%$ & 0.206 & & \\
\hline
\end{tabular}

F- One Way Analysis of Variance; Post HOC test Bonferroni [Compared control-ve group and the rest of the groups] p-value $>0.05 \mathrm{NS}$; *p-value $<0.05 \mathrm{~S} ; * *$ p-value $<0.001 \mathrm{HS}$

This table shows statistically significant difference between groups according to the number of vacuolated epithelial cells. When comparing the mean number of vacuolated epithelial cells, within the buccal mucosa epithelium, between all groups; +ve control $G p$ showed the significantly highest mean number of vacuolated epithelial cells, while the $\boldsymbol{P} \boldsymbol{\&} \boldsymbol{I F} \boldsymbol{G} \boldsymbol{p}$ showed the lowest mean number of vacuolated epithelial cells (Fig. 4).

TABLE (2): Comparison between groups according to No. of PCNA +ve cells.

\begin{tabular}{|c|c|c|c|c|c|c|c|c|}
\hline \multirow{2}{*}{ Groups } & \multicolumn{5}{|c|}{ No. of PCNA +ve cells } & \multicolumn{3}{|c|}{ ANOVA test } \\
\hline & Min. & Max. & Mean & $\pm \mathrm{SD}$ & Change\% & Bonferroni & $\mathrm{F}$ & p-value \\
\hline Control -ve Group & 65 & 101 & 82.22 & 11.56 & & --- & \multirow{5}{*}{57.579} & \multirow{5}{*}{$<0.001 * *$} \\
\hline Control +ve Group & 8 & 45 & 18.11 & 11.33 & -77.97 & $<0.001 * *$ & & \\
\hline Propolis Group & 56 & 96 & 75.44 & 12.36 & -8.25 & 0.261 & & \\
\hline Intermittent Fasting Group & 48 & 90 & 69.56 & 14.44 & -15.40 & $0.039^{*}$ & & \\
\hline Propolis + Intermittent Fasting Group & 88 & 132 & 104.56 & 13.08 & 27.17 & $<0.001^{* *}$ & & \\
\hline
\end{tabular}

F- One Way Analysis of Variance; Post HOC test Bonferroni [Compared control-ve group and the rest of the groups] p-value $>0.05 \mathrm{NS} ; *$ p-value $<0.05 \mathrm{~S} ; *$ *p-value $<0.001 \mathrm{HS}$

This table shows statistically significant difference between groups according to No. of PCNA +ve cells. When comparing the mean number of PCNA-positive cells, within the buccal mucosa epithelium, between all groups; +ve control $\boldsymbol{G p}$ showed the significantly lowest mean number of PCNA-positive cells, while $\boldsymbol{P} \boldsymbol{\&} \boldsymbol{I F} \boldsymbol{G} \boldsymbol{p}$ possessed the highest mean number of PCNA-positive cells (Fig. 5). 


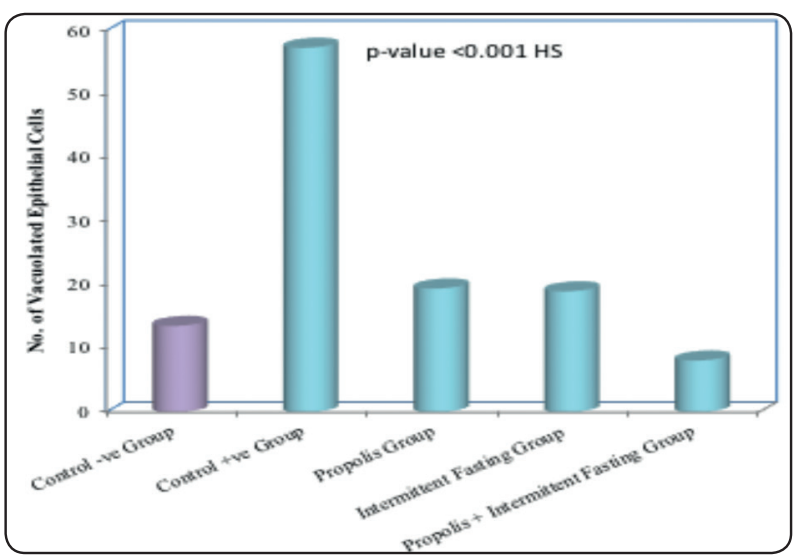

Fig. (4) Bar chart between groups according to No. of Vacuolated Epithelial Cells

\section{DISCUSSION}

In this study, the buccal mucosa was chosen as it is the most commonly affected oral region in cases of Oral Mucositis (OM). Moreover, inflammation \& ulceration in buccal mucosa usually compromises the patient's life style as it's an extremely painful condition ${ }^{[1,2,4]}$.

In this study, Propolis was of choice because many studies proved its anti-inflammatory $\&$ antioxidant effects ${ }^{[14-18]}$, and hence, it was appealing to test these abilities on healing oral ulcerations. Also, many of the medications available locally for oral mucositis are mainly anesthetic oral gels and can't cure inflammation. So, it would be of vast importance to create a drug that can actually treat the condition, not only masking it.

Many studies have recently proven that Intermittent Fasting (IF) boosted the immune system and reduced inflammation ${ }^{[19-24]}$. That's why it was so tempting to see the extent of its beneficial effects on OM. The protocol implemented in this study for IF was the "Time-Restricted Feeding", which is an IF protocol characterized by an eating arrangement in which food consumption is allowed in not more than 8 hours or less, daily. This protocol does not lead to the reduction of caloric input, instead, it focuses on the importance of well-defined feeding periods alternating with long fasting periods,

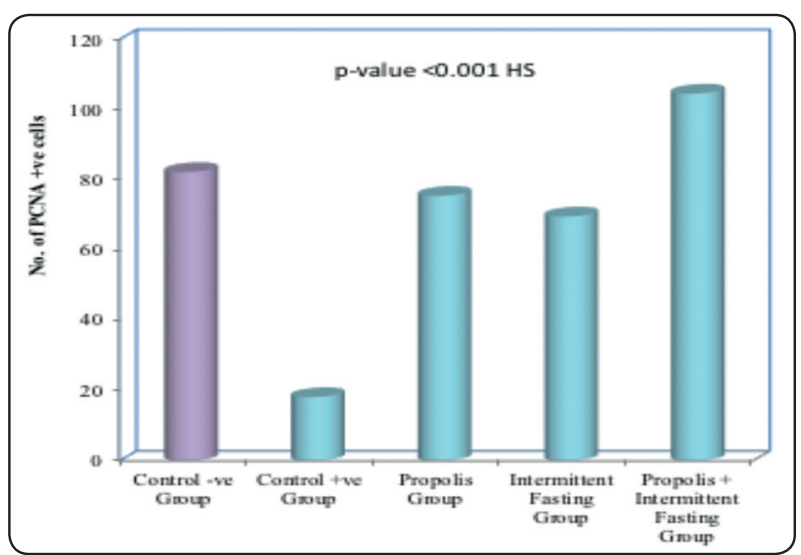

Fig. (5) Bar chart between groups according to No. of PCNA +ve cells.

daily. Since the amount of food is unlimited, this protocol can also be used in long-term experiments ${ }^{[28]}$. Moreover, in this study, the combined effect of both Propolis (P) \& Intermittent Fasting (IF) was investigated to obtain maximum benefits in treating $\mathrm{OM}$.

This study had investigated the effect of P \& IF by $\mathbf{H \& E}$ as a routine histologic examination to detect structural changes in both epithelium \& C.T in all groups.

LM examination of the $\mathbf{H \& E}$-stained sections of the +ve control Gp, showed noticeable thinning and sometimes disruption of the keratin layer overlying the epithelium. This finding could be due to interference with the cellular activities as protein synthesis, as a result of the underlying inflammatory process ${ }^{[4]}$. The $\mathbf{H} \boldsymbol{\&} \mathbf{E}$-stained sections of this group also displayed marked intra-epithelial cell vacuolations, which was statistically significant, when compared to the -ve control Gp. This finding could be attributed to hydropic degeneration of the epithelial cells secondary to apoptotic changes resulting from increased reactive oxygen species (ROS) production due to the underlying inflammatory process. The C.T of this group's specimens, showed marked extracellular edema, dilated engorged blood vessels, inflammatory cell infiltration and hyalinization of collagen fibers in 
many regions. These changes could be explained by the fact that transcription factors such as nuclear factor $-x \mathrm{~B}$ and others are activated during inflammation, and upregulate genes that control synthesis of inflammatory cytokines. Hence, activate enzymes that increase the rate of apoptosis, and enhance collagen destruction through the activation of Matrix Metalloproteinases (MMPs) ${ }^{[4]}$.

Histochemical examination of the +ve control Gp specimens, using Masson's Trichrome special stain, showed apparently more abundance of mature (appeared red) collagen fiber bundles relative to the -ve control Gp, with rarely detected immature fibers (appeared blue), and that indicated reduced regenerative capacity of the $\mathrm{CT}$ fibroblasts as a result of the previously mentioned inflammatory chemical changes in the tissue. This came in agreement with Atyade, et al, (2018) ${ }^{[32]}$ who found that, after experimental arthritis induction, the inflammatory infiltrate in the synovial membrane was rich in CD68 macrophages, CD3 lymphocytes, and CD20 lymphocytes. They also stated that the activated macrophages produced many toxic substances as nitric oxide, ROS and MMPs, destroying various types of collagen within the tissue. At the same time, these substances affected fibroblasts hindering their capacity to synthesize new collagen. Meanwhile, examination of the Anti-PCNA- stained specimens of the +ve control $\mathrm{Gp}$, revealed the presence of apparently fewer positive cells in the basal and parabasal layers of the epithelium as compared to the -ve control $\mathrm{Gp}$, which was also statistically confirmed. This pointed out the markedly reduced proliferation potential of the epithelium of this group. Again, that could be explained mainly by the accumulation of inflammatory end-products which might be deleterious for the actively-dividing basal epithelial cells, rendering them incapable to perform their function properly, and that came in agreement with Mohsen, et al ${ }^{[31]}$. Collectively, the previous findings outlined the ulcerative lesions in OM.
LM examination of the $\mathbf{H} \& \mathbf{E}$ stained sections of the $\mathbf{P}$ Gp, revealed great improvement in the epithelium and C.T, which was expressed by significant reduction in the mean value of vacuolated epithelial cells. Apparent increase in the immature collagen fibers relative to the mature ones in the C.T of this $\mathrm{Gp}$, which was evident in Masson's Trichrome stained specimens. This Gp also showed significantly increased epithelial cell proliferation, which was statistically proven by the significant increase in the mean number of Anti-PCNA positive cells, when compared to the + ve control Gp. All these findings collectively confirmed the ameliorating potential of Propolis, and particularly pointed out its anti-inflammatory potential, and that might explain that the C.T improvement in this Gp's specimens, was greater than that in the epithelium. This matched what came in many previous researches, as ${ }^{[8,13,15-18]}$. In one of these researches, Araujo, et al ${ }^{[13]}$ stated that the antiinflammatory properties of Propolis was studied in different models of acute and chronic inflammation, such as formaldehyde-induced arthritis and paw edema induced by $\mathrm{PGE}_{2}$, or radiation, as well as in acute inflammation induced by zymosan. In these studies, Propolis showed similar effects to that of anti-inflammatory drugs used as positive controls in the experiments.

Meanwhile, LM examination of the $\mathbf{H} \boldsymbol{\& E}$ stained sections of the IF Gp showed considerable improvement in the histological appearance of both epithelium and CT, manifested as significant reduction in the mean number of vacuolated epithelial cells, apparent increase in the immature collagen fibers relative to the mature ones in the CT as detected by Masson's Trichrome. This Gp also presented enhanced epithelial cell proliferation, which was statistically confirmed by the significant increase in the mean number of Anti-PCNA positive cells, when compared to the + ve control $\mathrm{Gp}$. These findings emphasized the marked effects of IF on improving the histological image of this 
Gp's specimens, and enhancing the healing process, which came in agreement with many previous researches ${ }^{[19-25,29]}$.

Martin, et al ${ }^{[19]}$ stated that Data from the animal studies described in their review showed that neurons in the brains of rats and mice subjected to IF, exhibited greater resistance to oxidative, metabolic and excitotoxic insults. Moreover, Tikoo, et al ${ }^{20]}$ confirmed in their study that IF caused significant improvement in blood urea, nitrogen, creatinine, albumin and HDL cholesterol, parameters that are associated with the development of diabetic nephropathy. In their study, diabetic rats on IF also displayed significant improvement in onset of hypertension.

Anton, et al [22] reported that IF caused additional biological changes that may contribute to the observed enhancement in health and endurance of persons on IF, including enhanced cellular quality control through autophagy ("self-eating" of damaged organelles), and the maintenance of a healthy population of mitochondria though production of new mitochondria. That could be the best explanation for the great reduction in epithelial cell vacuolations, and in the enhanced proliferating activity of epithelium and regeneration of C.T in this study, of the IF group.

The IF\&P Gp presented a histological \& immune-histochemical image which greatly resembled that of the -ve control Gp. H\&E-stained sections of this $\mathrm{Gp}$ revealed significant lowering of mean values of vacuolated epithelial cells, marked reduction or even rarity of inflammatory cells and extracellular edema in CT. Regeneration of collagen fibers was proven by Masson's Trichrome special stain which revealed that the majority of collagen fibers were immature (new collagen formation) indicating enhanced healing. This Gp also revealed significantly increased Anti-PCNA positive cells by immunohistochemical examination, which was confirmed statistically, indicating markedly increased proliferating capacity of the epithelium. The findings displayed by this Gp's specimens greatly emphasized the huge role of both Propolis and IF, especially when combined together, in ameliorating the inflammatory changes associated with OM. And since no one had attempted to study the combined effect of both P\&IF before (up to our knowledge), it was very encouraging to see their results in this study.

\section{REFERENCES}

1. Nair G R, Naidu GS, Jain S, Nagi R, Makkad R S and Jha A. Clinical Effectiveness of Aloe Vera in the Management of Oral Mucosal Diseases- A systematic Review. J Clin Diag Res. 2016;10(8): ZE01-ZE07.

2. Espinoza I, Rojas R, Arand W, Jamonal J. Prevalence of oral mucosal lesions in elderly people in Santiago, Chile. J Oral Pathol Med. 2003 Nov; 32(10): 571-5.

3. Baharvand $\mathbf{M}$, Jafari $\mathrm{S}$ and Mortazavi H. Herbs in oral mucositis. J Clin Diag Res. 2007 Mar; 11(3): ZE05-ZE11.

4. Debra J Harris. Cancer Treatment-Induced Mucositis Pain: Strategies for Assessment and Management. Ther Clin Risk Manag. 2006 sep;2(3):251-258.

5. Thomsen M and Vitetta L. Adjunctive treatments for the prevention of chemotherapy -and-radiotherapy- Induced mucositis. Intgr Cancer Ther. 2018 Dec; 17(4):1027-1047.

6. Osama M. Maria, Nicoletta E., Muanza T. Radiation -Induced Oral Mucositis. Front Oncol. 2017 May 22; 7:89.

7. Vanita and Varun Suri. Menopause and Oral Health. MidLife Health. 2014,vol 5, Issue 3, Pg 115-120.

8. Hajialyani M., Tewari D., Eduardo S., Mohammad S., Mohammad F. Natural Product-Based Nanomedicine. 2018 sep, 3;13:5023-5043.

9. Salehi B., Lopez P., Pons-Foster E., Calina D., Sharifi M., Ramirez K., et al. Plant-derived Bioactives in Oral Mucosal Lesions: A Key Emphasis to Curcumin, Lycopene, Chamomile, Aloe Vera, Green Tea. Biomolecules. 2019 Mar 17;9(3):106.

10. Saraswathi G. and Amala M. Review on Aloe Vera- the Plant of Marvel in the Field Medicine and Dentistry. Int Jour of Current Res . 2017 Feb, Vol 9, Issue 02,pp 4665346657. 
11. Raeessi M, Raeessi R, Panahi Y, Gharaie H, Masoud S, Saadat A, et al. "Coffee Plus Honey" Versus "Topical Steroids" in the Treatment of Chemotherapy-Induced Oral Mucositis. Complement. Altern Med. 2014 Aug 8;14:293.

12. Jaouni S., Muhayawi M, Hussein A, Elfiki I, Al-Raddadi R, Al Masaudi S, et al.. Effects of Honey on Oral Mucositis Among Pediatric Cancer Patients Undergoing Chemo/ Radiotherapy Treatment at King Abdul Aziz University Hospital in Jeddah, Kingdom of Saudi A

13. Araujo M, Liberio S, Guerra R, Ribeiro M, Nascimento F. Mechanisms of Action Underlying the Anti-Inflammatory and Immunomodulatory Effects of Propolis. Rev. bras. farmacogen. Vol. 22 no. 1.2012 Feb.

14. Demelo A, Almeida L, Sancho M, Mate A. Composition and Properties of Apis Mellifera Honey. Journal of Api cultural Research. 2017.

15. Kocot J, Kielczykowska M, Kocot D, Kurzepa J, and Musik I. Antioxidant Potential of Propolis, Bee Pollen, and Royal Jelly: Possible Medical Application. Oxid Med Cell Longev. 2018;2018:7074209.

16. Silva R, Machado B, Barreto G, Costa S, Andrade R, et al. Antioxidant, antiseptic and cytotoxic properties of various Brazilian propolis extracts. PLoS One. 2017; 12(3):e0172585.

17. Khacha-anada S, Tragoolpua K, Chankawannakul P, Tragoolpua Y.Antioxidant and anti-cancer cell proliferation activity of propolis extracts from two extraction methods. Asian Pac J Cancer Prev. 2013, vol 14 (11),6991-6995.

18. Marcucci M, Ferreres F, Viguera C, Bankova V, Castro S, Dantas A, et al. Phenolic Compounds from Brazilian Propolis with Pharmacological Activities. Journal of Ethnopharmacology, Vol 74, Issue 2, Feb 2001. Pg 105-112.

19. Martin B, Mattson M, Maudsley S. Caloric Restriction and Intermittent Fasting: Two Potential Diets for Successful Brain Aging. Aging Res Rev. 2006; 5 (3):332-353.

20. Tikoo K, Tripathi D, Kabara D, Sharma V, Gaikwad A. Intermittent Fasting Prevents the Progression of Type I Diabetic Nephropathy in Rats. FEBS Lett. 2007 Mar 6; 581 (5):1071-8.

21. Smith C, Mayer J and Duncan I. Autophagy Promotes Oligodendrocyte Survival and Function Following Dysmyelination in a Long-Lived Myelin Mutant. J Neuro Sci. 2013 May 1;33 (18): 8088-8100.
22. Anton S, Leeuwenburgh C. Fasting or Caloric Restriction for Healthy Aging. Exp Gerontol. 2013 Oct; 48(10):10031005 .

23. Steinkraus K, Smith E, Davis C, Pendergrass W, Sutphin $\mathrm{G}$ and Kennedy B, et al. Dietary Restriction Suppresses Proteotoxicity and Enhances Longevity by an hsf-1dependent Mechanism in Caenorhabditis Elegans. Aging Cell. 2008, Vol 7, Issue 3, Pg 394-404.

24. Madorsky I, Opalach K, Waber A, Verrier J, Solmo $\mathrm{C}$, Foster $\mathrm{T}$, et al. Intermittent Fasting Alleviates the Neuropathic Phenotype in a Mouse Model of CharcotMarie-Tooth Disease. Neurobiol Dis. 2009 Apr; 34 (1): 146-154.

25. Wegman M, Guo M, Bennion D, Shankar M, Chrzanowski S, Goldberg, et al. Practicality of Intermittent Fasting in Humans and its Effects on Oxidative Stress and Genes Related to Aging and Metabolism. Rejuvenation Res. 2015 Apr; 18(2) : 162-72.

26. Aral C, Kesim S, Greenwell H, Kara M, Cetin A and Yakan B. Alveolar Bone Protective and Hypoglycemic Effects of Systemic Propolis Treatment in Experimental Periodontitis and Diabetes Mellitus. Journal of Medicinal Food, Vol 18, no. 2, 2015.

27. Viet C, Corby P, Akinwande A, Schmidt B. Review of Preclinical Studies on Treatment of Mucositis and Associated Pain. Journal of Dental Research. 2014. Vol 93, Issue 9, Pages: 868-875.

28. Maze Engineers. How to Model Intermittent Fasting in Rodents. Conduct Science, 2019.

29. Ahmet I, Wan R, Mattson M, Lakatta E, Talan M. Cardioprotection by Intermittent Fasting in Rats. CirculationAHA. 2005;112:3115-3121.

30. Bancroft J, Gamble M. Theory \& Practice of Histological Techniques. 2008.

31. Mohsen R, Halawa A, Hassan R. Role of Bone MarrowDerived Stem Cells Versus Insulin on Filiform and Fungiform Papillae of Diabetic Albino Rats (Light, Fluorescent and Scanning Electron Microscopic Study). Acta Histochemica. 2019 (121) : 812-822.

32. Atyade S, Velosa A, Catanozi S, Bianco V, Andrade P and De Castro J, et al. Collagen V Oral Administration Decreases Inflammation and Remodelling of Synovial Membrane in Experimental Arthritis. PLOS ONE. 2018. 